\title{
Impact of Dose Reduction on Efficacy: Implications of Exposure-Response Analysis of Palbociclib
}

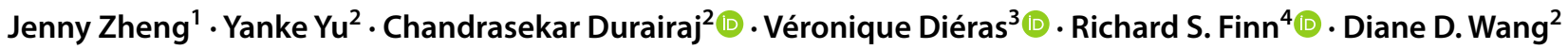

Published online: 19 November 2020

(c) The Author(s) 2020

\begin{abstract}
Background Palbociclib is indicated for hormone receptor-positive, human epidermal growth factor receptor 2-negative advanced breast cancer (ABC).

Objective Exposure-response analyses were conducted to evaluate efficacy in Asian versus non-Asian patients and in patients with versus without dose reduction in PALOMA-2.

Patients and Methods PALOMA-2 compared palbociclib plus letrozole versus placebo plus letrozole in patients with ABC. Population pharmacokinetic analysis provided apparent palbociclib clearance (CL/F) for each patient. The time-varying

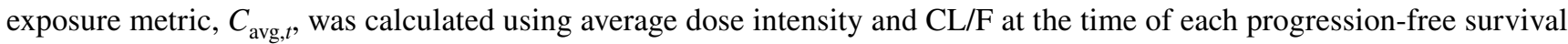
(PFS) event. A Cox proportional model characterized PFS and palbociclib $C_{\text {avg }, t}$ relationships. Significant prognostic factors for PFS were identified by univariate analysis, which were subsequently included in multivariate analyses, in addition to the $C_{\text {avg, }, t}$ effect on PFS. PFS profiles in Asian/non-Asian patients and patients with/without dose reduction were simulated and compared using observed palbociclib exposures and established exposure-response relationships.

Results Patients $(n=421)$ received palbociclib plus letrozole (Asian $=64$, non-Asian $=357$; no dose reduction $=272$, dose reduction =149). Based on univariate analyses, significant prognostic factors were Ki67 score, age, and baseline aspartate aminotransferase (BAST), tumor size, alkaline phosphatase, and albumin levels. In multivariate analysis, only Ki67 and BAST remained significant. Palbociclib exposure did not significantly affect PFS in either univariate $(P=0.12)$ or multivariate $(P=0.44)$ analyses.

Conclusions This analysis suggests that palbociclib exposure has no impact on PFS when the dose reduction algorithm from palbociclib clinical trials is used. There is no difference in efficacy between Asians and non-Asians, despite the higher level of dose reductions in Asians.
\end{abstract}

Pfizer NCT01740427.

\section{Key Points}

Palbociclib dose reduction does not affect efficacy of the drug.

Although Asian patients experienced greater dose reduction, palbociclib efficacy was not compromised.

Jenny Zheng

jenny.zheng@pfizer.com

1 Pharmacometrics, Global Product Development, Pfizer Inc, Col-D/006/D6400, 500 Arcola Rd., Collegeville, PA 19426, USA

2 Clinical Pharmacology, Global Product Development, Pfizer Inc, San Diego, CA, USA

3 Department of Medical Oncology Institut Curie, Paris and Centre Eugène Marquis, Rennes, France

4 David Geffen School of Medicine at UCLA, Santa Monica, CA, USA

\section{Introduction}

Breast cancer is the most common cancer among women worldwide, with $\sim 2.1$ million cases reported in 2018 [1]. Treatment choice for breast cancer is decided based on several factors, including the status of hormone (estrogen and progesterone) receptors and human epidermal growth factor receptor 2 (HER2) expression. Endocrine therapy is the backbone for initial management of patients with hormone receptor-positive $(\mathrm{HR}+)$ advanced breast cancer. However, patients eventually develop resistance to such treatments [2-4]; therefore, new treatments are needed for those patients.

PALOMA-2 is a phase 3 , international, multicenter, randomized, double-blind, placebo-controlled trial designed to investigate whether palbociclib plus letrozole provides superior clinical benefit compared with letrozole plus placebo in postmenopausal women with estrogen receptor-positive, HER2 - advanced breast cancer who had not received any 
prior systemic therapy for their advanced disease [5]. The study results demonstrated a statistically significant, robust, and clinically meaningful 10-month increase in median progression-free survival (PFS), the primary endpoint, with palbociclib plus letrozole versus placebo plus letrozole (24.8 vs. 14.5 months; hazard ratio, 0.58 [95\% confidence interval (CI) 0.46-0.72]; one-sided $P<0.000001$ ) [5].

The recommended palbociclib starting dose is $125 \mathrm{mg}$ once daily (QD) taken with food for 21 days, followed by 7 days off treatment. The palbociclib dose is recommended to be interrupted or reduced based on individual safety and tolerability. The trial showed that overall efficacy of palbociclib plus letrozole was superior to placebo plus letrozole, even though $36 \%$ of palbociclib-treated patients required dose interruption/reduction to manage toxicity [5]. However, the question remains whether patients who required a dose reduction had inferior efficacy compared with patients who did not have dose reduction. In addition, dose reductions were more common in Asian patients compared with non-Asian patients [6]. The impact on efficacy of the increased frequency of dose reduction in Asian compared with non-Asian patients needs to be evaluated. The objective of this exposure-response analysis is to evaluate the effect of palbociclib exposure changes (i.e., dose reduction) on PFS.

\section{Materials and Methods}

\subsection{Study Design}

The details of the PALOMA-2 study design, patient population, and study assessments have been previously published [5]. Briefly, 666 patients were randomly assigned in a 2:1 ratio to the palbociclib plus letrozole group or to the placebo plus letrozole group. Patients assigned to the palbociclib plus letrozole group received palbociclib $125 \mathrm{mg}$ orally QD from day 1 to day 21, followed by 7 days off treatment, during each 28-day cycle in combination with continuous oral letrozole $2.5 \mathrm{mg}$ QD. Those assigned to the placebo plus letrozole group received placebo orally from day 1 to day 21 , followed by 7 days off treatment, during each 28 -day cycle in combination with matching letrozole.

The study was conducted in compliance with the ethical principles originating in or derived from the Declaration of Helsinki and in compliance with all International Council on Harmonisation Good Clinical Practice guidelines. The final protocol, any amendments, and informed consent documentation were reviewed and approved by the Institutional Review Board and/or Independent Ethics Committee at each of the investigational centers participating in the study.

\subsection{Pharmacokinetic Assessments and the Exposure Variable}

Pharmacokinetic (PK) samples were collected at hour 0 (predose) on day 14 of cycle 1 and cycle 2 from all patients. In a subset of patients participating in the intensive electrocardiogram (ECG) assessment substudy, plasma PK samples were collected at $0 \mathrm{~h}$ (pre-dose) and 2, 4, 6, and $8 \mathrm{~h}$ after palbociclib plus placebo dosing on day 14 of cycle 1 . Blood samples were processed, and plasma palbociclib concentrations were determined using high-performance liquid chromatography tandem mass spectrometry as described previously [7]. The linearity of the calibration curve for palbociclib was in the range of $1-250 \mathrm{ng} / \mathrm{mL}$, and the lower limit of quantification for the palbociclib assay was $1.00 \mathrm{ng} / \mathrm{mL}$.

Pharmacokinetic concentrations were analyzed using a population PK (PPK) approach, and the individual apparent clearance (CL/F) values obtained from the PPK analysis were used to calculate the individual palbociclib exposures used in the exposure-response analysis. To account for changes in palbociclib exposure over time resulting from dose interruption or reduction, the individual time-varying average palbociclib concentration values $\left(C_{\text {avg }, t, i}\right)$ were calculated at the time of each PFS event $(t)$ for all patients who were still at risk based on the corresponding daily dose intensity calculated as the cumulative dose in $\mathrm{mg}$ up to time $t$ divided by treatment duration in days and individual CLi/F, using the following equation:

$C_{\text {avg }, t, i=} \frac{\text { Daily dose intensity up to } t}{\mathrm{CL}_{i} / F}$

\subsection{Efficacy Assessments and Response Variables}

The primary efficacy endpoint, investigator-assessed PFS, was used in the exposure-response analysis. The efficacy assessment details have been published previously [5].

\subsection{Palbociclib Exposure Versus Progression-Free Survival}

Data from only the palbociclib plus letrozole group were used in the exposure-response analysis. To graphically explore whether PFS is dependent on palbociclib concentration, the distribution of palbociclib concentration, i.e., 5th, 50th (median), and 95th percentiles of $C_{\mathrm{avg}, t}$ in all patients who were at risk and the palbociclib $C_{\text {avg, } t}$ values in patients with PFS were compared at the time of each event. A trend that palbociclib $C_{\text {avg, } t}$ in patients with PFS was below the median would suggest that PFS might be exposure dependent.

A Cox proportional model was used to quantify the relationship between PFS and $C_{\text {avg, } t}$. The metastatic site (i.e., 
visceral and nonvisceral) was included in the model as a strata factor. Univariate analysis was used to screen and identify prognostic factors. The prespecified covariates, in addition to $C_{\text {avg, },}$, were age; body weight; baseline tumor size (i.e., the sum of the longest diameters of non-nodal lesions and short axis for nodal lesions, per RECIST guidelines); Eastern Cooperative Oncology Group performance status; disease-free interval; baseline lab values, including albumin, alkaline phosphatase, aspartate aminotransferase (AST), total bilirubin, and absolute neutrophil count; biomarkers, including cyclin D1 amplification, p16 loss, retinoblastoma copies ( $\mathrm{Rb}$ ), estrogen receptor (ER), and Ki67 expression. Assessment of ER, Ki67, p16, and Rb scores was performed based on a protocol described previously [8]. Multivariate analysis was used to account for the potential confounding effects of the prognostic factors. Identified significant covariates from the univariate analysis, in addition to $C_{\text {avg }, t}$, were simultaneously included in the multivariate analysis. The model that included all significant covariates in the multivariate analysis and $C_{\text {avg, } t}$ was considered the final model. Likelihood ratio tests were used to examine the significance of the covariate, and a $P$ value of $\leq 0.05$ was used in both univariate and multivariate analyses.

\subsection{Evaluation of the Effect of Dose Reduction on Progression-Free Survival}

The Cox proportional model of the multivariate analysis was used to simulate PFS profiles for a "typical" patient who would have received the full palbociclib dose and a "typical" patient who would have received the reduced dose; the PFS profiles were then compared. For concentration, the median $C_{\text {avg, } t}$ versus time in each group of interest was calculated and used in the simulation. For all other prognostic factors, the median values of the prognostic factors were used.

Patients who had received a palbociclib dose $<125 \mathrm{mg}$ at any time were assigned to the dose-reduction group. Otherwise, patients were assigned to the no-dose-reduction group. In each group, the median palbociclib concentration values for patients still in the trial at the time of each event were calculated and used as $C_{\mathrm{avg}, t}$ for simulating PFS profiles in the "typical" patient in the group, while the other prognostic factors used in the simulation were fixed to be the median values of the prognostic factors obtained from the trial. The "typical" patients were assumed to have nonvisceral disease.

\subsection{The Progression-Free Survival Comparisons in Asian and Non-Asian Patients}

Patients were stratified into Asian and non-Asian groups; the median values of average dose intensity and $C_{\mathrm{avg}, t}$ for patients who were still in the trial at the time of each PFS event were calculated in both groups and presented graphically. The PFS profiles were simulated based on the median $C_{\mathrm{avg}, t}$ versus time in each group, while all other prognostic factors were fixed as the median values of those prognostic factors observed in the trial. A nonvisceral disease site was assumed for both Asian and non-Asian patients.

\section{Results}

\subsection{Patient Disposition and Demographics}

A total of 666 patients were randomly assigned in a 2:1 ratio to the palbociclib plus letrozole group (444 patients) or to the placebo plus letrozole group (222 patients). Among the 444 patients in the palbociclib plus letrozole group, 23 patients did not have PK data and, therefore, were not included in the exposure-response analysis. The baseline demographic characteristics are presented in Table 1.

The 5th, 50th (median value), and 95th percentile of palbociclib concentrations for all patients and the palbociclib concentrations for patients who had PFS at the time of each event are presented in Fig. 1. The red circles, which represent the $C_{\mathrm{avg}, t}$ for patients who had PFS, are distributed around the median concentration. It appeared there were slightly more red circles below the median concentration at the event times, suggesting a trend that patients who had PFS had lower palbociclib exposure. Univariate analysis showed that $C_{\mathrm{avg}, t}$ is not significantly associated with PFS. Ki67 score, age, baseline AST level, alkaline phosphatase level, and albumin value were significantly associated with PFS (Table 2).

In multivariate analysis, age, alkaline phosphatase level, and albumin values were no longer significant, but Ki67 score and baseline AST level were found to be associated with PFS (Table 3). After accounting for the potential confounding effects of the prognostic factors identified from univariate analysis, palbociclib exposure remained unassociated with PFS $(P=0.44)$. The effect of $C_{\mathrm{avg}, t}$ on PFS was reevaluated when Ki67 and baseline AST level were included in the analysis, with the resultant findings indicating that palbociclib exposure is not associated with PFS $(P=0.69)$.

Among 421 patients included in the exposure-response analysis, 272 patients were in the no-dose-reduction group and 149 patients were in the dose-reduction group, including 88 patients with doses reduced to $100 \mathrm{mg}$ and 61 patients with doses reduced to $75 \mathrm{mg}$. Simulated PFS curves for the "typical" patients in both groups are presented in Fig. 2, with similar results observed between the two groups.

Among the 421 patients in the analysis, 64 were Asian and 357 were non-Asian. The median values of palbociclib average dose intensity and concentration over time for Asian and non-Asian patients are presented in Fig. 3. In Asians and non-Asians, mean $\pm \mathrm{SD}$ average dose intensity was 
Table 1 Baseline covariates for patients in exposure-response analysis

\begin{tabular}{|c|c|c|c|}
\hline Variables & $n^{\mathrm{a}}$ & Mean (SD) & Median (min-max) \\
\hline Age, years & 421 & $61.70(10.68)$ & $62.00(30.00-89.00)$ \\
\hline Baseline albumin, $\mathrm{mg} / \mathrm{dL}$ & 421 & $4.07(0.41)$ & $4.10(2.80-5.20)$ \\
\hline Baseline alkaline phosphatase, U/L & 421 & $125.72(109.44)$ & $94.00(17.00-1125.00)$ \\
\hline Baseline absolute neutrophil count, $10^{6} / \mathrm{mL}$ & 421 & $3.97(1.66)$ & $3.66(1.45-12.31)$ \\
\hline Baseline AST, U/L & 421 & $28.69(15.95)$ & $25.00(3.00-153.00)$ \\
\hline Baseline total bilirubin, $\mathrm{mg} / \mathrm{dL}$ & 421 & $0.50(0.20)$ & $0.50(0.07-1.40)$ \\
\hline Baseline tumor size, $\mathrm{mm}^{\mathrm{b}}$ & 322 & $53.44(40.93)$ & $42.00(10.00-258.00)$ \\
\hline Body weight, $\mathrm{kg}$ & 421 & $70.47(16.65)$ & $67.95(34.50-156.80)$ \\
\hline CCND1 score, $\%$ & 358 & $110.84(59.44)$ & $120.00(0.00-275.00)$ \\
\hline ER score, $\%^{\mathrm{c}}$ & 361 & $111.14(75.88)$ & $120.00(0.00-290.00)$ \\
\hline Ki67 score, $\%^{\mathrm{c}}$ & 351 & $24.98(23.12)$ & $20.00(0.00-95.00)$ \\
\hline P16 score, $\%^{\mathrm{c}}$ & 348 & $55.47(63.23)$ & $30.00(0.00-295.00)$ \\
\hline $\mathrm{Rb}$ score, $\%^{\mathrm{c}}$ & 357 & $73.25(56.40)$ & $65.00(0.00-270.00)$ \\
\hline Variable name and group & & & Number of patients \\
\hline \multicolumn{4}{|l|}{ Progression-free survival } \\
\hline Event & & & 180 \\
\hline Censored & & & 241 \\
\hline \multicolumn{4}{|l|}{ Disease site } \\
\hline Visceral & & & 208 \\
\hline Non-visceral & & & 213 \\
\hline \multicolumn{4}{|l|}{ Disease interval } \\
\hline De novo advanced disease & & & 139 \\
\hline$\leq 12$ months since completion of prior (nec & & & 83 \\
\hline$>12$ months since completion of prior (nec & & & 199 \\
\hline \multicolumn{4}{|l|}{ ECOG PS } \\
\hline 0 & & & 243 \\
\hline 1 & & & 169 \\
\hline 2 & & & 9 \\
\hline \multicolumn{4}{|l|}{ Race } \\
\hline Caucasian & & & 323 \\
\hline Black & & & 8 \\
\hline Asian & & & 64 \\
\hline Other & & & 26 \\
\hline
\end{tabular}

AST aspartate aminotransferase, CCND1 cyclin D1, ECOG PS Eastern Cooperative Oncology Group performance status, ER estrogen receptor, Max maximal value, Min minimal value, $R b$ retinoblastoma, RECIST Response Evaluation Criteria in Solid Tumors

${ }^{a} n$ values reflect the number of patients for whom the corresponding covariate was available for analysis

${ }^{b}$ Baseline tumor size was defined as the sum of the longest diameters of non-nodal lesions and short axis for nodal lesions, per RECIST guidelines

${ }^{\mathrm{c}} \mathrm{ER}, \mathrm{Ki} 67$, p16, and Rb scores were assessed following a protocol that was described previously [8]

$75.9 \pm 15.9$ and $84.8 \pm 14.3 \mathrm{mg} /$ day, respectively. Despite the lower dose intensity in Asian patients as a result of more frequent dose reduction compared with non-Asian patients, the average palbociclib concentration was higher in Asian than in non-Asian patients $\left(C_{\text {avg }, t}\right.$ was $102.4 \pm 36.7$ and $82.8 \pm 31.6 \mathrm{ng} / \mathrm{mL}$, respectively), which is attributed to the observation that Asian patients had lower plasma CL/F than non-Asian patients (33.6 vs. $48.0 \mathrm{~L} / \mathrm{h}$ ). Simulated PFS curves in Asian and non-Asian patients are presented in Fig. 4, with similar profiles observed for Asian and nonAsian patients. 


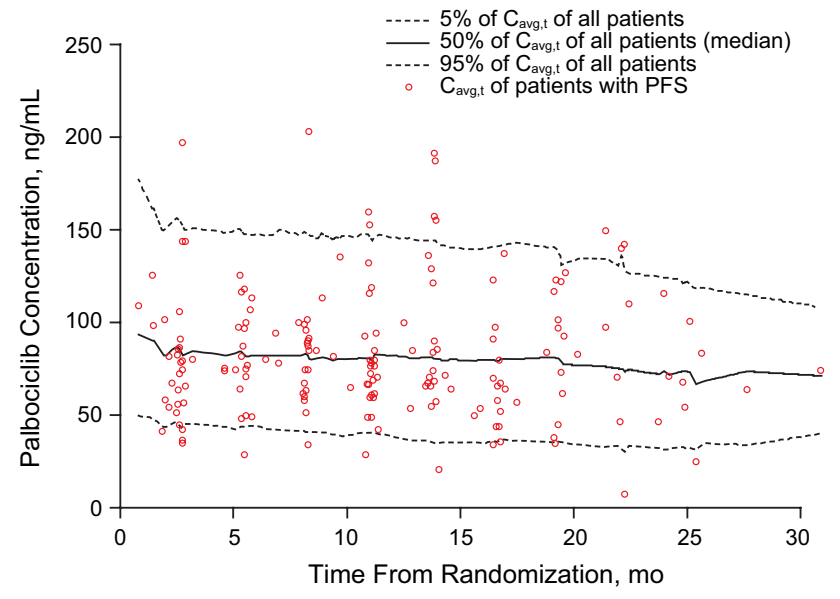

Fig. 1 Palbociclib concentration distribution at time of each event. $C_{a v g, t}$ time-varying average concentration. PFS progression-free survival

\section{Discussion}

Exposure-response analyses have been routinely conducted for oncology drugs as a way to evaluate doses used in clinical trials $[9,10]$. The findings from the analysis can be used to inform dose selection in clinical trials and to evaluate efficacy in situations in which drug exposures are changed (e.g., in special populations or owing to drug-drug interactions). Palbociclib is approved for treatment of adult patients with HR+, HER2- advanced or metastatic breast cancer in combination with either an aromatase inhibitor as initial endocrine-based therapy in postmenopausal women or in men, or fulvestrant in patients with disease progression following endocrine therapy $[11,12]$. To better manage the safety of the drug, palbociclib doses were reduced as necessary in the clinical trial setting $[5,13]$. The current exposureresponse analyses evaluate the potential impact on efficacy due to differences in palbociclib exposure between patients with dose reduction and those with no dose reduction, and between Asian and non-Asian patients.

Table 2 Significant covariates from univariate analysis of Cox proportional model

\begin{tabular}{|c|c|c|c|c|}
\hline & $n^{\mathrm{a}}$ & Coefficient & Hazard ratio & $P$ value \\
\hline$C_{\text {avg }, t}, \mathrm{ng} / \mathrm{mL}$ & 421 & -0.00377 & 0.996355 & 0.1175 \\
\hline Ki67 score, $\%$ & 351 & 0.0123 & 1.012 & 0.0002 \\
\hline Age, years & 421 & -0.0195 & 0.981 & 0.004 \\
\hline Baseline AST, U/L & 421 & 0.0101 & 1.010 & 0.0233 \\
\hline Baseline tumor size, $\mathrm{mm}^{\mathrm{b}}$ & 322 & 0.0048 & 1.005 & 0.024 \\
\hline Baseline alkaline phosphatase, U/L & 421 & 0.0013 & 1.001 & 0.0307 \\
\hline Baseline albumin, $\mathrm{mg} / \mathrm{dL}$ & 421 & -0.3820 & 0.682 & 0.0483 \\
\hline
\end{tabular}

$A S T$ aspartate aminotransferase, $C_{a v g, t}$ time-varying average concentration, RECIST Response Evaluation Criteria in Solid Tumors ${ }^{a} n$ values reflect the number of patients for whom the corresponding covariate was available for analysis

${ }^{b}$ Baseline tumor size was defined as the sum of the longest diameters of non-nodal lesions and short axis for nodal lesions, per RECIST guidelines

Table 3 Multivariate analysis of Cox proportional model $(N=277)$

\begin{tabular}{lrll}
\hline Variable & Coefficient & Hazard ratio (95\% CI) & $P$ value \\
\hline$C_{\text {avg }, t}$, ng/mL & -0.00224 & $0.998(0.992-1.003)$ & 0.440 \\
Ki67 expression, \% & 0.00889 & $1.009(1.002-1.016)$ & 0.013 \\
Age, years & -0.01334 & $0.987(0.971-1.002)$ & 0.097 \\
Baseline AST, U/L & 0.01102 & $1.011(1.002-1.02)$ & 0.016 \\
Baseline tumor size, mm ${ }^{\mathrm{a}}$ & 0.00369 & $1.004(0.999-1.008)$ & 0.111 \\
Baseline albumin, mg/dL & -0.17757 & $0.837(0.532-1.318)$ & 0.443 \\
Baseline alkaline phosphatase, U/L & 0.00048 & $1.000(0.999-1.002)$ & 0.490 \\
\hline
\end{tabular}

AST aspartate aminotransferase, $C_{\text {avg }, t}$ time-varying average concentration, RECIST Response Evaluation Criteria in Solid Tumors

${ }^{a}$ Baseline tumor size was defined as the sum of the longest diameters of non-nodal lesions and short axis for nodal lesions, per RECIST guidelines 


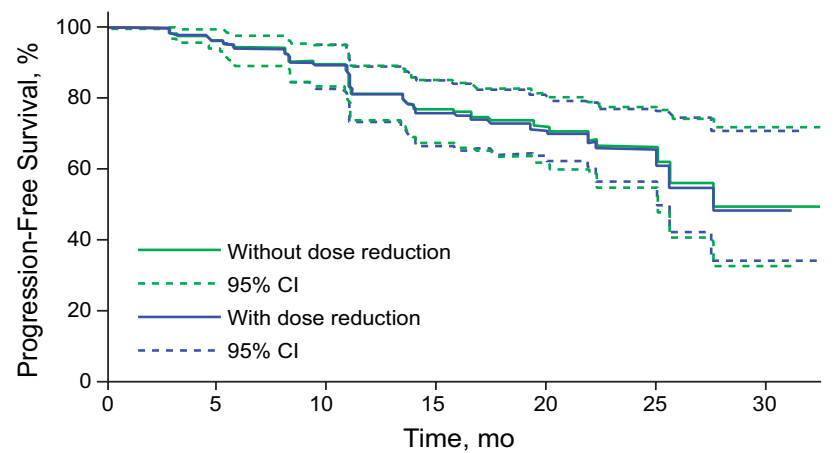

Fig. 2 Simulated progression-free survival for patients with and without dose reduction
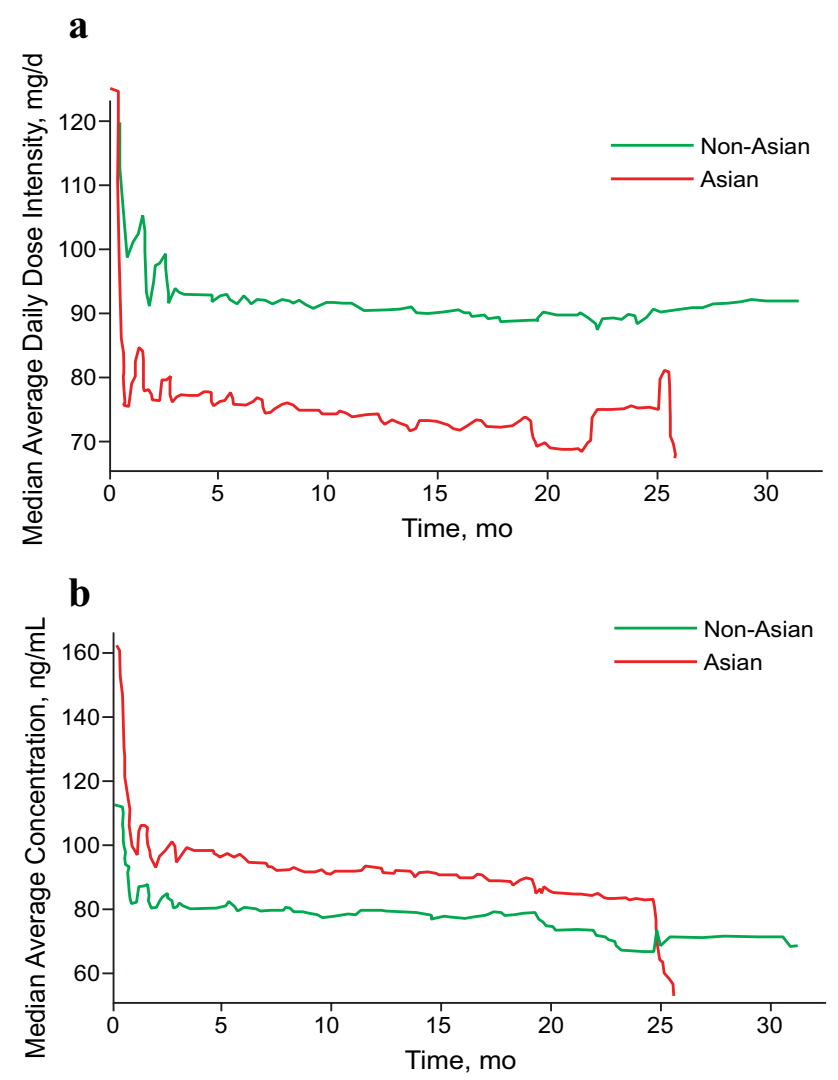

Fig. 3 Palbociclib average dose intensity (a) and concentration (b) vs. time in Asian and non-Asian patients

The most intuitive way of evaluating the effect of dose reduction during treatment is to compare PFS in patients who had dose reduction versus those who did not. However, such a direct comparison could be biased owing to the confounding effect between dose reduction and time to PFS, that is, patients who had longer PFS time would have a higher probability of having dose reduction [14-16]. Similarly,

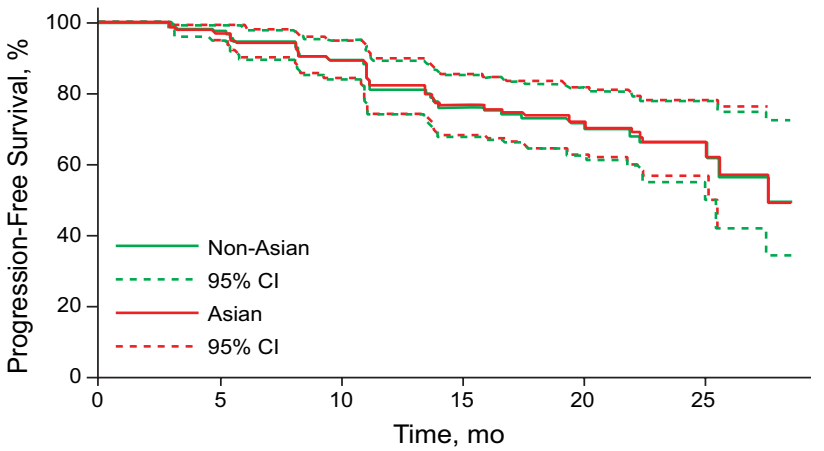

Fig. 4 Simulated progression-free survival in Asian and non-Asian patients

a single palbociclib exposure during the entire treatment duration, $C_{\text {avg }}$, for each individual was not an appropriate exposure metric in this exposure-response analysis because $C_{\text {avg }}$ is calculated based on time to PFS: patients who had longer PFS would have a higher probability of having dose reduction, and thus lower $C_{\text {avg }}$. To account for the changes in palbociclib exposure over time, the time-varying exposure metric $C_{\text {avg }, t}$ was correlated with PFS. In this time-varying covariate analysis, the correlation between PFS and palbociclib exposure was evaluated at the time of each event based on individual average palbociclib exposure and the covariate values for all patients who were at risk at the time.

Because the study was randomized at the treatment level instead of the exposure level, drug exposure could be confounded with other prognostic factors. It is critical that the potential confounding effects of other prognostic factors with palbociclib exposure be accounted for in the exposure-response analysis. Univariate analyses were used to identify prognostic factors, and multivariate analysis was used to estimate palbociclib exposure effect when the other prognostic factors were accounted for. The analyses showed that even after accounting for the effects of other prognostic factors, such as age, Ki67 score, baseline AST level, baseline alkaline phosphatase level, baseline tumor size, and baseline albumin level, PFS was not associated with palbociclib exposure. Subsequently, the model was used in a simulation to evaluate whether PFS differences resulted from palbociclib exposure changes alone. The findings suggested that the PFS profiles are similar in patients with and without dose reduction. The advantage of the approach is that the effect of dose reduction on PFS can be evaluated after removing the effect of other potential confounding factors, assuming the exposure-response relationship has been reasonably well defined.

It is important to understand the assumptions of this analysis. First, a linear relationship was assumed between covariates, including palbociclib exposure, and the log (hazard) 
based on the equation, $\log (\lambda)=\log \left(\lambda_{0}\right)+\beta_{1} * C_{\text {avg }, t}+\beta_{2}$ $* \operatorname{Cov}_{1}+\cdots+\beta_{n} * \operatorname{Cov}_{n}$. This assumption is reasonable because we often do not have sufficient data to quantify a nonlinear relationship. However, we must be cautious not to extrapolate the relationship. Second, the treatment effect of palbociclib does not change over time; that is, the effect of dose reduction is the same regardless of when dose reduction occurred. Third, since letrozole exposure was not measured in this trial, the impact of letrozole on PFS and its interaction with palbociclib were not assessed. Finally, this is a post hoc exploratory analysis; the study design was not ideal for identifying a robust exposure-response relationship.

It has been reported that the neutrophil response to palbociclib treatment is exposure related [17]. Assuming that time to PFS is also related to palbociclib exposure, a patient who has low clearance and therefore high drug exposure would have a longer PFS but would be more likely to develop neutropenia, which may lead to dose reduction. The dose-reduction algorithm would narrow the exposure range, thereby reducing the power to identify the exposureresponse relationship. The robustness of the evaluation of the impact on efficacy due to exposure difference, estimated via simulation, depends on the robustness of the original exposure-response relationship. The effect of dose reduction on the evaluation of the exposure-response relationship remains to be studied.

Even though dose reduction was more common in Asian than non-Asian patients in the PALOMA-2 study [6], the palbociclib exposures in Asian patients were higher, not lower, than in non-Asian patients because Asian patients had lower palbociclib CL/F. The simulation showed similar PFS for Asian and non-Asian patients.

In summary, exposure-response analysis can be used to evaluate the effect of exposure changes on efficacy. Our analysis suggests that the dose-reduction algorithm implemented in the palbociclib trial would not have a significant effect on PFS in patients with dose reduction. Moreover, the PFS outcome between Asian and non-Asian patients was similar even though Asian patients had a higher incidence of dose reduction [6]. It is important to note that the palbociclib dose-modification algorithms should not be changed beyond what the product label suggests.

Acknowledgements This study was sponsored by Pfizer Inc. Editorial support was provided by Jennifer Fetting, Ph.D., of ICON plc (North Wales, PA, USA), and was funded by Pfizer Inc.

\section{Declarations}

Funding This study was sponsored by Pfizer Inc.

Conflicts of interest V.D. has served as a consultant/advisor for Pfizer, Roche, Genentech, Eli Lilly, Novartis, AbbVie, AstraZeneca, MSD, Daiichi Sankyo, and Seattle Genetics; served on the speakers bu- reau for Pfizer, Roche, Eli Lilly, AstraZeneca, Daiichi Sankyo, and Novartis; and received travel, accommodation, and expenses from Roche, Novartis, Pfizer, Eli Lilly, and AstraZeneca. R.S.F. has a consultant advisory role with Pfizer, Bayer, Novartis, and Bristol Myers Squibb and receives research funding from Pfizer. J.Z., Y.Y., C.D., and D.D.W. are employees and shareholders of Pfizer.

Ethics approval All procedures performed in studies involving human participants were in accordance with the ethical standards of the institutional and/or national research committee and with the 1964 Helsinki declaration and its later amendments or comparable ethical standards.

Consent to participate For this type of study, formal consent is not required.

Consent for publication For this type of study, formal consent is not required.

Availability of data and materials Upon request, and subject to certain criteria, conditions and exceptions (see https://www.pfizer.com/scien ce/clinical-trials/trial-data-and-results for more information), Pfizer will provide access to individual de-identified participant data from Pfizer-sponsored global interventional clinical studies conducted for medicines, vaccines, and medical devices (1) for indications that have been approved in the USA and/or EU or (2) in programs that have been terminated (i.e., development for all indications has been discontinued). Pfizer will also consider requests for the protocol, data dictionary, and statistical analysis plan. Data may be requested from Pfizer trials 24 months after study completion. The de-identified participant data will be made available to researchers whose proposals meet the research criteria and other conditions, and for which an exception does not apply, via a secure portal. To gain access, data requestors must enter into a data access agreement with Pfizer.

Code availability N/A.

Author contributions V.D, R.S.F., J.Z., Y.Y., C.D., and D.D.W. participated in the study design, data collection, and analysis and interpretation of data. J.Z. wrote the first draft of the manuscript. All authors provided significant input into the manuscript and have read and approved the final draft for submission.

Open Access This article is licensed under a Creative Commons Attribution-NonCommercial 4.0 International License, which permits any non-commercial use, sharing, adaptation, distribution and reproduction in any medium or format, as long as you give appropriate credit to the original author(s) and the source, provide a link to the Creative Commons licence, and indicate if changes were made. The images or other third party material in this article are included in the article's Creative Commons licence, unless indicated otherwise in a credit line to the material. If material is not included in the article's Creative Commons licence and your intended use is not permitted by statutory regulation or exceeds the permitted use, you will need to obtain permission directly from the copyright holder. To view a copy of this licence, visit http://creativecommons.org/licenses/by-nc/4.0/.

\section{References}

1. Bray F, Ferlay J, Soerjomataram I, et al. Global cancer statistics 2018: GLOBOCAN estimates of incidence and mortality worldwide for 36 cancers in 185 countries. CA Cancer J Clin. 2018;68(6):394-424. 
2. Mouridsen H, Gershanovich M, Sun Y, et al. Superior efficacy of letrozole versus tamoxifen as first-line therapy for postmenopausal women with advanced breast cancer: results of a phase III study of the International Letrozole Breast Cancer Group. J Clin Oncol. 2001;19(10):2596-606.

3. Smith IE, Dowsett M. Aromatase inhibitors in breast cancer. N Engl J Med. 2003;348:2431-42.

4. Chia S, Gradishar W, Mauriac L, et al. Double-blind, randomized placebo controlled trial of fulvestrant compared with exemestane after prior nonsteroidal aromatase inhibitor therapy in postmenopausal women with hormone receptor-positive, advanced breast cancer: results from EFECT. J Clin Oncol. 2008;26(10):1664-70.

5. Finn RS, Martin M, Rugo HS, et al. Palbociclib and letrozole in advanced breast cancer. N Engl J Med. 2016;375(20):1925-36.

6. Im SA, Mukai H, Park IH, et al. Palbociclib plus letrozole as first-line therapy in postmenopausal Asian women with metastatic breast cancer: results from the phase III, randomized PALOMA-2 study. J Glob Oncol. 2019;5:1-19.

7. Tamura K, Mukai H, Naito Y, et al. Phase I study of palbociclib, a cyclin-dependent kinase 4/6 inhibitor, Japanese patients. Cancer Sci. 2016;107(6):755-63.

8. Finn RS, Liu Y, Zhu Z, et al. Biomarker analyses of response to cyclin-dependent kinase 4/6 inhibition and endocrine therapy in women with treatment-naive metastatic breast cancer. Clin Cancer Res. 2020;26(1):110-21.
9. Agrawal S, Feng Y, Roy A, Kollia G, Lestini B. Nivolumab dose selection: challenges, opportunities, and lessons learned for cancer immunotherapy. J Immunother Cancer. 2016;4:72.

10. Yang J, Zhao H, Garnett C, et al. The combination of exposureresponse and case-control analyses in regulatory decision making. J Clin Pharmacol. 2013;53(2):160-6.

11. IBRANCE ${ }^{\circledR}$ capsules (palbociclib). Full prescribing information. New York, NY, USA: Pfizer Inc; 2019.

12. IBRANCE ${ }^{\circledR}$ (palbociclib). Summary of product characteristics. Sandwich, Kent, UK: Pfizer Limited; 2018.

13. Turner NC, Ro J, Andre F, et al. Palbociclib in hormonereceptor-positive advanced breast cancer. N Engl J Med. 2015;373(3):209-19.

14. Anderson JR, Cain KC, Gelber RD. Analysis of survival by tumor response. J Clin Oncol. 1983;1(11):710-9.

15. Buyse M, Piedbois P. On the relationship between response to treatment and survival time. Stat Med. 1996;15(24):2797-812.

16. Suissa S. Immortal time bias in pharmaco-epidemiology. Am J Epidemiol. 2008;167(4):492-9.

17. Sun W, O'Dwyer PJ, Finn RS, et al. Characterization of neutropenia in advanced cancer patients following palbociclib treatment using a population pharmacokinetic-pharmacodynamic modeling and simulation approach. J Clin Pharmacol. 2017;57(9):1159-73. 